\section{Estimation of Shape of Binary Polygonal Object from Scattered Field}

\author{
A. Buvaneswari and Prabhakar S. Naidu
}

\begin{abstract}
We present a method of estimating the shape of a weakly scattering binary convex polygonal object from the backscattered field. We use the concept scattering centers where it has been possible to model the scattered field as a sum of complex sinusoids. All corners of an object could be estimated with three or more illuminations, out of which at least two are in orthogonal directions. Using the amplitude of the sinusoids, we have also resolved the ambiguity in joining the corners when there are more than one object.
\end{abstract}

Index Terms-Broadband illumination, diffraction tomography, pairing algorithm, shape estimation.

\section{INTRODUCTION}

If the boundary of an object is piecewise linear, the corner points are sufficient for pattern recognition, image compression and coding, and shape analysis, etc. [1]-[6]. The presently available corner detection algorithms work on spatial image data, that is, a photograph. The present work is aimed at corner detection from the scattered wavefield (acoustic or electromagnetic). When an object

Manuscript received December 8, 1995; revised March 4, 1997. The associate editor coordinating the review of this manuscript and approving it for publication was Prof. Ken D. Sauer.

The authors are with the Department of Electrical and Computer Engineering, Indian Institute of Science, Bangalore 560012, India (e-mail: psn@ece.iisc.ernet.in).

Publisher Item Identifier S 1057-7149(98)01015-X.

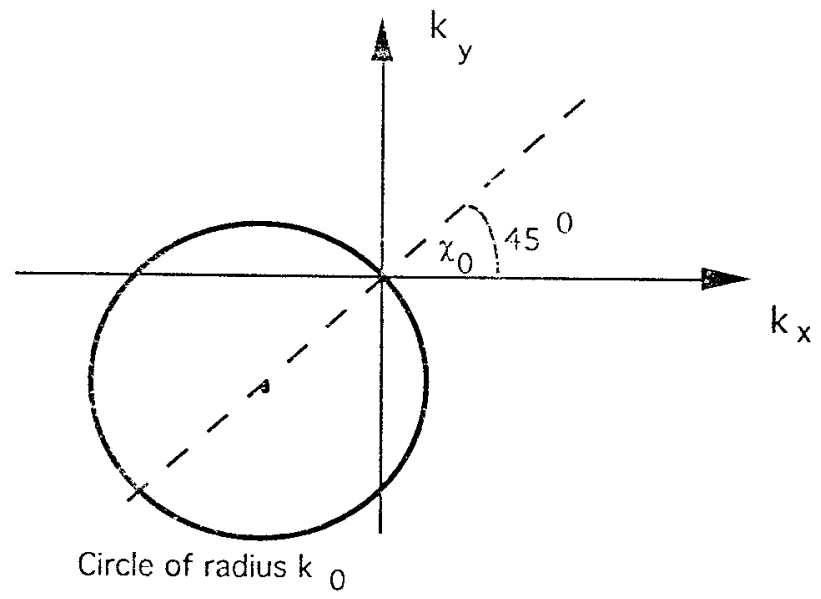

Fig. 1. For an angle of incidence, $\chi_{0}=45^{\circ}$, the scattered field received by a circular array is proportional to the object Fourier transform on a circle as shown.

whose refractive index is slightly different with respect to that of the surrounding medium is illuminated with a plane wave, the scattered field measured around the object is proportional to the Fourier transform of the object. Thus, the shape information is buried in the scattered field. It is of some interest in medical diagnosis, in subsurface imaging, and in nondestructive testing to be able to recognize the shape of the buried object from the scattered acoustic or electromagnetic field, particularly when only a few limited views are permitted. We shall show (Section II) that when the object is binary, convex, and having a nondegenerate polygonal cross section, the scattered field is a sum of sinusoids, a function of wave number and corners of the polygon. The object is illuminated with a broadband plane wave and the scattered field is measured as a function of wavenumber. The frequencies of the sinusoids are estimated from the scattered field using an algorithm described in [7] and [8] (Section IV). The approach has been extended to a multiobject scene in Section V, where we shall assume that the number of objects present is known; only shape and location are unknown. Finally, some computer simulation results are presented in Section VI.

Though we consider only the cases where the spatial Fourier data is obtained through DT imaging, the mathematical results and the ideas presented in this work can be well utilized for even straight line tomography or in any other imaging modality that provides spatial Fourier information.

\section{DifFRACTION TOMOGRAPHY}

Diffraction tomography refers to the cross-sectional imaging of objects from diffracted or scattered wavefield [9], [10]. An object is illuminated from various directions with a diffracting source of energy such as acoustic waves, whose wavelength is comparable with the scale of inhomogeneities. The incident wave energy is scattered in all directions by diffraction process. Usually, a long linear array of sensors facing the incident wave field is used to record the forward scatter, but the backscatter is lost. A circular array transceivers was proposed to capture both forward and backward scatter [11]. It is shown in [11] that the scattered field measured by a circular array is proportional to two-dimensional (2-D) Fourier transform of the object profile taken on the circumference of a circle of radius equal to the wave number and centered at $\left(-k_{0} \cos \chi_{0},-k_{0} \sin \chi_{0}\right)$, where $\chi_{0}$ 


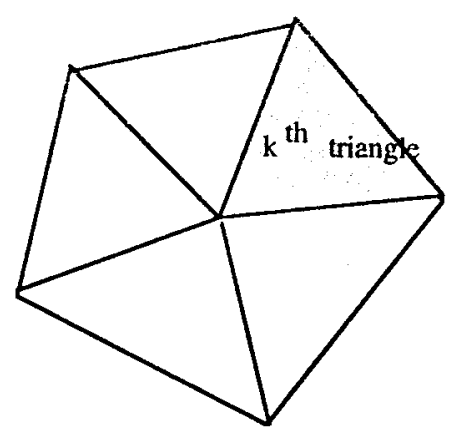

(a)

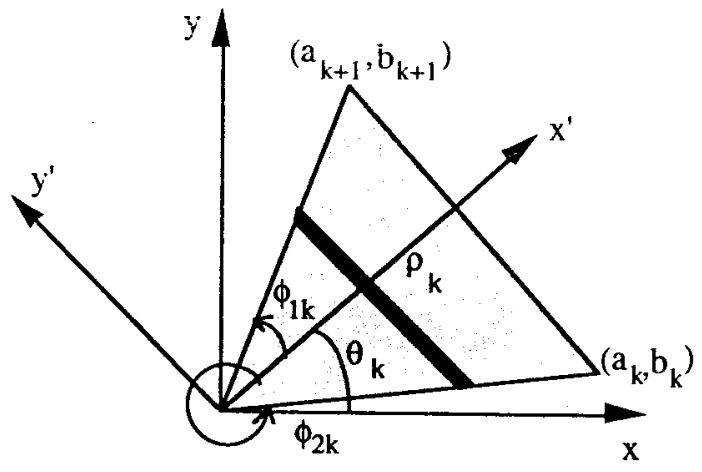

(b)

Fig. 2. To evaluate the Fourier transform of a polygonal object, we consider each triangle.

is the angle of illumination (see Fig. 1). By changing the direction of illumination $\left(0\right.$ to $\left.360^{\circ}\right)$, the object Fourier transform is scanned over a disk of radius $k_{0}$. By inverse Fourier transformation, we can reconstruct the object profile pixel by pixel. When the object is binary (i.e., refractive index is constant through out the object), the interest is in the shape of the object. The shape information may be directly obtained from the scattered field without having to reconstruct the object profile pixel by pixel. This approach was taken by Milanfar and co-workers [12], [13] in the context of ray tomography, where the input data is projections of the object.

\section{Fourier Transform of a Binary Convex Polygonal ObJect}

Consider the evaluation of the 2-D Fourier transform over a $p$ sided binary and convex polygonal domain (see Fig. 2). Take any point inside the polygon and join it to all corners forming $p$ triangles, which lie entirely inside the polygon and make this as the origin of the coordiante system:

$$
\begin{aligned}
F\left(k_{x}, k_{y}\right) & =\iint_{\text {over polygon }} e^{j\left(k_{x} x+k_{y} y\right)} d x d y \\
& =\sum_{n=1}^{p} \iint_{\text {over }} e_{\text {thiangle }}^{j\left(k_{x} x+k_{y} y\right)} d x d y .
\end{aligned}
$$

To evaluate the integral over $n$th triangle, refer to Fig. 2(b), where we show the integration along a narrow strip under a rotated coordinate system such that the new $x$-axis is perpendicular to $n$th side. Note that this is valid for a class of nondegenerate objects that are star-shaped with respect to the origin (convex bodies form a subset of these). The triangle is then covered by a series of strips. Equation (1) reduces to

$$
\begin{aligned}
F\left(k_{x}, k_{y}\right)= & \sum_{n=1}^{p} \int_{0}^{\rho_{n}} \int_{x^{\prime} \tan \phi_{2 n}}^{x^{\prime} \tan \phi_{1 n}} \\
& e^{-j\left(\left(k_{x} \cos \theta_{n}+k_{y} \sin \theta_{n}\right) x^{\prime}+\left(k_{y} \cos \theta_{n}-k_{x} \sin \theta_{n}\right) y^{\prime}\right)} d y^{\prime} d x^{\prime}
\end{aligned}
$$

where $x^{\prime}=\left(x \cos \theta_{k}+y \sin \theta_{k}\right)$ and $y^{\prime}=\left(y \cos \theta_{k}-x \sin \theta_{k}\right)$. Evaluate the integral in (2) first with respect to $y^{\prime}$ followed by integration with respect to $x^{\prime}$. We obtain

$$
\begin{aligned}
F\left(k_{x}, k_{y}\right)= & \sum_{n=1}^{p} \frac{\left[e^{-j\left(k_{x}^{\prime}-k_{y}^{\prime} \tan \phi_{1 n}\right) \rho_{n}}-1\right]}{k_{y}^{\prime}\left(k_{x}^{\prime}-k_{y}^{\prime} \tan \phi_{1 n}\right)} \\
& -\frac{\left[e^{-j\left(k_{x}^{\prime}-k_{y}^{\prime} \tan \phi_{2 n}\right) \rho_{n}}-1\right]}{k_{y}^{\prime}\left(k_{x}^{\prime}-k_{y}^{\prime} \tan \phi_{2 n}\right)}
\end{aligned}
$$

where $k_{x}^{\prime}=\left(k_{x} \cos \theta_{n}+k_{y} \sin \theta_{n}\right)$ and $k_{y}^{\prime}=\left(k_{y} \cos \theta_{n}-k_{x} \sin \theta_{n}\right)$. We shall now rewrite (3) by replacing $\theta_{n}, \phi_{1 n}$, and $\phi_{2 n}$ in terms of the coordinates of the two corners corresponding to the $n$th side, namely, $\left(a_{n}, b_{n}\right)$ and $\left(a_{n+1}, b_{n+1}\right)$. The following relations are used for this purpose:

$$
\begin{aligned}
\rho_{n} & =a_{n} \cos \theta_{n}+b_{n} \sin \theta_{n} \\
\rho_{n} & =a_{n+1} \cos \theta_{n}+b_{n+1} \sin \theta_{n} \\
a_{n} & =\rho_{n}\left(\cos \theta_{n}+\sin \theta_{n} \tan \phi_{1 n}\right) \\
b_{n} & =\rho_{n}\left(\sin \theta_{n}-\cos \theta_{n} \tan \phi_{1 n}\right) \\
a_{n+1} & =\rho_{n}\left(\cos \theta_{n}-\sin \theta_{n} \tan \phi_{2 n}\right) \\
b_{n+1} & =\rho_{n}\left(\sin \theta_{n}+\cos \theta_{n} \tan \phi_{2 n}\right) .
\end{aligned}
$$

We obtain

$$
\begin{aligned}
& F\left(k_{x}, k_{y}\right) \\
& \quad=\sum_{n=1}^{p} \rho_{n}\left[\frac{e^{-j\left(k_{x} a_{n}+k_{y} b_{n}\right)}-1}{k_{y}^{\prime}\left(k_{x} a_{n}+k_{y} b_{n}\right)}-\frac{e^{-j\left(k_{x} a_{n+1}+k_{y} b_{n+1}\right)}-1}{k_{y}^{\prime}\left(k_{x} a_{n+1}+k_{y} b_{n+1}\right)}\right] .
\end{aligned}
$$

Our goal is to determine $\left(a_{n}, b_{n}\right)$ and $\left(a_{n+1}, b_{n+1}\right)$ from (4). This may be achieved by expressing (4) on the $k_{y}=0$ and $k_{x}=0$ axes. We get following equations:

$$
\begin{aligned}
& k_{x}^{2} F\left(k_{x}, k_{y}=0\right) \\
&=-\sum_{n=1}^{p} \rho_{n}\left\{\frac{e^{-j k_{x} a_{n}}-1}{a_{n} \sin \theta_{n}}-\frac{e^{-j k_{x} a_{n+1}}-1}{a_{n+1} \sin \theta_{n}}\right\} \\
& k_{y}^{2} F\left(k_{y}=0, k_{y}\right) \\
&=\sum_{n=1}^{p} \rho_{n}\left\{\frac{e^{-j k_{x} b_{n}}-1}{b_{n} \sin \theta_{n}}-\frac{e^{-j k_{x} b_{n+1}}-1}{b_{n+1} \sin \theta_{n}}\right\} .
\end{aligned}
$$

The above equations may be solved by modeling them as a sum of sinusoids and using the well-known Prony's algorithm or its more modern versions [7], [8]. From the coefficients in the exponents of the complex sinusoids we obtain $\left(a_{n}, a_{n+1}\right)$ and $\left(b_{n}, b_{n+1}\right)$, but we are yet to pair them, that is, select the right $x$ and $y$ coordinate pair, which will form a valid corner.

\section{PAIRING ALgORITHM}

In the previous section, we saw how to obtain the $x$ and $y$ coordinates of the corners of the polygon. This alone will not suffice to define a unique convex polygon. We need some additional information on how to pair a given $x$-coordinate with the right $y$ coordinate from the list of estimated $y$-coordinates. This problem was resolved by using an additional illumination at an angle $\theta$

$$
\begin{aligned}
k^{2} F(k \cos \theta, k \sin \theta)= & \sum_{n=1}^{p} \Gamma_{n}\left\{\frac{\left[e^{-j k\left(a_{n} \cos \theta+b_{n} \sin \theta\right)}-1\right]}{\left(a_{n} \cos \theta+b_{n} \sin \theta\right)}\right. \\
& \left.-\frac{\left[e^{-j k\left(a_{n+1} \cos \theta+b_{n+1} \sin \theta\right)}-1\right]}{\left(a_{n+1} \cos \theta+b_{n+1} \sin \theta\right)}\right\} .
\end{aligned}
$$


TABLE I

$x$ AND $y$ COORDINATES AND THEIR LINEAR Combination $\left(\theta=30^{\circ}\right)$ Are Shown in the Table

\begin{tabular}{c|c|c}
\hline$x$ & $y$ & $x \cos \theta+y \sin \theta$ \\
\hline 9.0981 & 6.0981 & 10.9282 \\
\hline 3.9019 & 9.0981 & 7.9282 \\
\hline 0.9019 & 3.9019 & 2.7321 \\
\hline 6.0981 & 0.9019 & 5.7321 \\
\hline
\end{tabular}

TABLE II

Estimated Projections from the Scattered Field (Noise Free) Are Shown in the Figure

\begin{tabular}{|c|c|}
\hline $\mathrm{x}$ & $9.0981,6.0981,3.9019,0.9019$ \\
\hline y & $9.0981,6.0981,3.9019,0.9019$ \\
\hline$x \cos \theta+y \sin \theta$ & $10.9282, \quad 5.7321,7.9282, \quad 2.7321$ \\
\hline
\end{tabular}

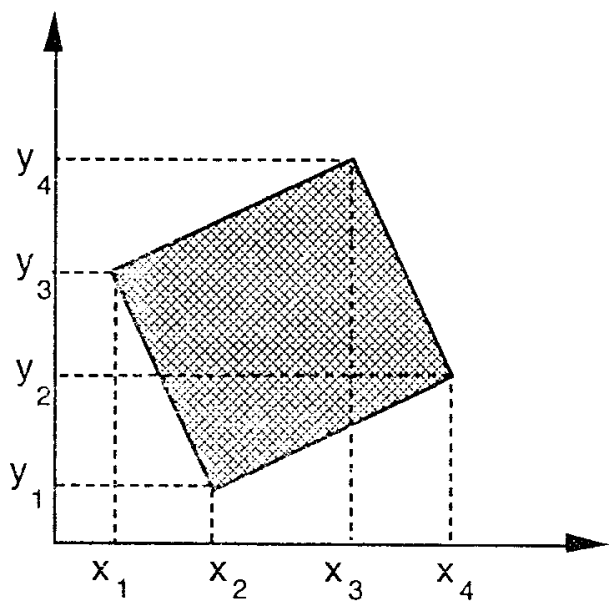

Fig. 3. Square object and the projection of its corners on the $x$ and $y$ axes.

$\Gamma_{n}=\frac{\rho_{n}}{\sin \left(\theta-\theta_{n}\right)}$. From the backscatter due to an illumination at angle $\theta\left(\neq 0\right.$ or $\left.\frac{\pi}{2}\right)$, we can estimate the coefficients in the exponents of the complex sinusoids, as described in Section II. Thus, we get additional information in the form of linear combination of the $x$ and $y$ coordinates of the corners, $\left(a_{n} \cos \theta+b_{n} \sin \theta\right) n=1,2, \cdots p$. The steps in the pairing algorithm are as follows.

1) Generate a list of $x$-coordinates, $y$-coordinates, and the linear combination of the $x$ and $y$ coordinates. It is presumed that the list is not in the same order as indexed corners.

2) Take the first element from the $x$-coordinate list and anyone element from the $y$-coordinate list and form a linear combination, $\left(a_{1} \cos \theta+b_{n} \sin \theta\right) n=1,2, \cdots p$.

3) Compare the result of linear combination with those estimated with $\theta\left(\neq 0\right.$ or $\left.\frac{\pi}{2}\right)$ illumination. The best match (within the limits of estimation error) will indicate the correct choice of $b_{n}$.

4) Take the next element from the $x$-coordinate list and go to step (2).

For illustration purposes, we consider a square object of size $(6 \mathrm{~m}, 6 \mathrm{~m})$, rotated by $30^{\circ}$ and shifted away from the origin by $(5 \mathrm{~m}, 5 \mathrm{~m})$. It is illuminated from three directions, $0, \pi$, and $\pi / 6$. The $x$ and $y$ coordinates got from the noiseless scattered field got in the first two directions and their linear combination $\left(\theta=30^{\circ}\right)$ are shown in Table I, and those estimated from the scattered field got in the third direction are shown in Table II. The application of pairing algorithm is illustrated in Table III. The best match with the
TABLE III

Numerical Illustration of the Pairing Algorithm $\left(\theta=30^{\circ}\right)$

\begin{tabular}{c|c|c|c}
\hline $\mathrm{x}$ & $\mathrm{y}$ & $x \cos \theta+y \sin \theta$ & $\begin{array}{c}\text { Best match } \\
\text { for } \mathrm{x}\end{array}$ \\
\hline 9.0981 & 9.0981 & 12.4282 & 6.0981 \\
& 6.0981 & $\mathbf{1 0 . 9 2 8 2}$ & \\
& 3.9019 & 9.8302 & \\
\hline 6.0981 & 0.9019 & 8.3301 & \\
& 9.0981 & 9.8302 & 0.9019 \\
& 6.0981 & 8.3301 & \\
& 3.9019 & 7.2321 & \\
\hline 3.9019 & 9.9019 & 5.7321 & \\
& 6.0981 & 7.9282 & 9.0981 \\
& 3.9019 & 6.4290 & \\
& 0.9019 & 5.3301 & \\
\hline 0.9019 & 9.0981 & 5.3302 & \\
& 6.0981 & 3.8301 & 3.9019 \\
& 3.9019 & 2.7321 & \\
& 0.9019 & 0.5000 & \\
\hline
\end{tabular}

TABLE IV

Amplitudes of the Sinusoids ARe Compared with the Computed Amplitudes for Three Different Convex Models, Which May Be Obtained by Joining the Corners. The Model with the Best Match (a) Is the Actual Model

\begin{tabular}{c|c|c|c|c|c}
\hline $\begin{array}{c}\mathrm{X} \\
\text { coordinate } \\
\text { in meters }\end{array}$ & $\begin{array}{c}\mathrm{y} \\
\text { coordinate } \\
\text { in meters }\end{array}$ & $\begin{array}{c}\text { Ooscrved } \\
\text { amplitudes }\end{array}$ & $\begin{array}{c}\text { Computed } \\
\text { amplitudes } \\
\text { Model (a) }\end{array}$ & $\begin{array}{c}\text { Computed } \\
\text { amplitudes } \\
\text { Model (b) }\end{array}$ & $\begin{array}{c}\text { Computed } \\
\text { amplitudes } \\
\text { Model (c) }\end{array}$ \\
\hline-8.8451 & -6.793 & -3.112 & -3.112 & -3.112 & -3.112 \\
\hline-6.793 & -1.155 & 3.112 & 3.112 & 4.8914 & 2.0898 \\
\hline-3.207 & -8.8451 & 3.112 & 3.112 & 2.5084 & 3.112 \\
\hline-1.155 & -3.207 & -3.112 & -3.112 & -2.8895 & 0.7085 \\
\hline 0.9019 & 3.9019 & -2.3094 & -2.3094 & 1.7240 & -2.7989 \\
\hline 3.9019 & 9.0981 & 2.3094 & 2.3094 & 2.3094 & -3.1547 \\
\hline 6.0981 & 0.9019 & 2.3094 & 2.3094 & 1.1655 & 5.4640 \\
\hline 9.0981 & 6.0981 & -2.3094 & -2.3094 & -2.3094 & -2.3094 \\
\hline
\end{tabular}

estimated coefficients is shown in column three in bold figure and the corresponding $y$-coordinate is shown in the last column.

We may encounter the problem of repeated $x$ or $y$ coordinates or their projections. The projections of two corners may overlap or come very close to each other depending upon the orientation of the object. As shown in Fig. 3, for a square object depending upon the orientation, the adjacent projections (e.g. $x_{1}$ and $x_{2}$ ) may come close to each other or overlap. The problem of repeated projection can be resolved by selecting another set of $x, y$, and $\theta$ directions whenever the number of sinusoids estimated in $x, y$, and $\theta$ illuminations differ. The number of sinusoids observed in all three illuminations must be equal. In practice, it may be necessary to illuminate an unknown object along several directions and estimate the sinusoids along each direction. From this set, choose three directions, preferably two orthogonal directions, having equal number of sinusoids. The number of sinusoids that may be determined from a finite data in the presence of noise is indeed a complex problem and, hence, it is outside the scope of this paper. The reader may like to refer to a book, for example, [14], or current literature on this topic.

\section{Multiple ObJects}

When there are two or more objects, as the center of coordinates would lie outside all but one object, it is necessary to modify (4), which was derived under the assumption that the center lies inside the object. Also, there may be some ambiguity in the process of constructing the object shape even when all $x$ and $y$ coordinates are correctly paired. The amplitude of the sinusoid corresponding to a corner can then be used to resolve such an ambiguity in addition to the fact that the objects are convex and the number of objects is 


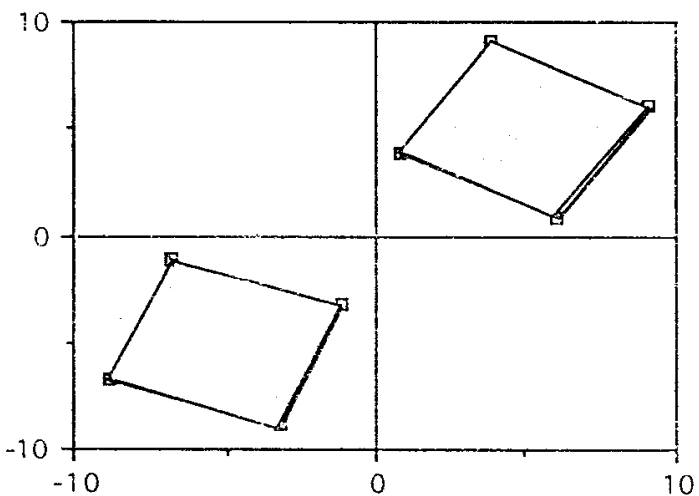

(a)

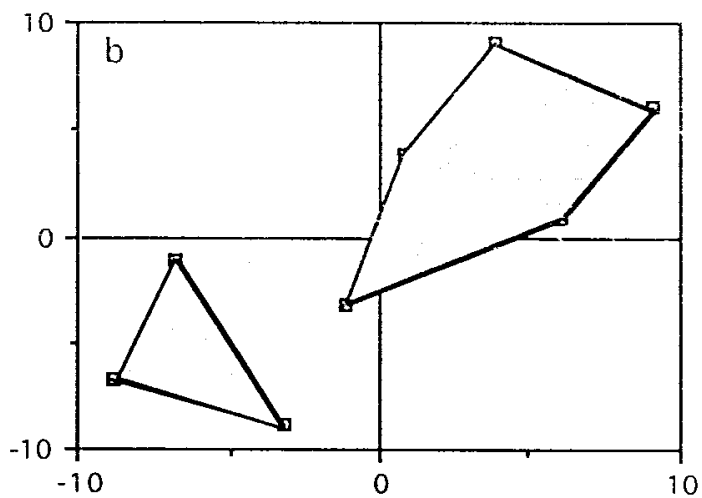

(b)

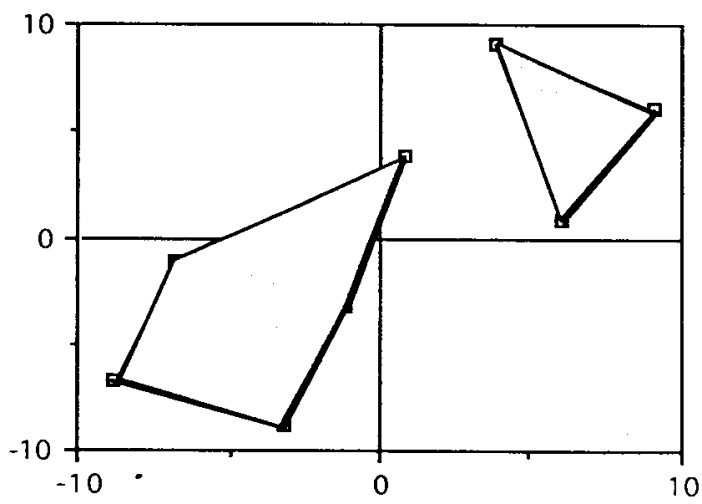

(c)

Fig. 4. Three different convex models can be obtained by joining the corners estimated from the scattered wavefield.

known. For this, we rewrite (6) as a sum of contributions from $p$ corners.

$$
k^{2} F(k \cos \theta, k \sin \theta)=\sum_{n=1}^{p} T_{n}\left\{e^{-j k\left(a_{n} \cos \theta+b_{n} \sin \theta\right)}-1\right\}
$$

where

$$
T_{n}=\frac{1}{\left(a_{n} \cos \theta+b_{n} \sin \theta\right)}\left\{\frac{\rho_{n}}{\sin \left(\theta-\theta_{n}\right)}-\frac{\rho_{n-1}}{\sin \left(\theta-\theta_{n-1}\right)}\right\}
$$

or, after simplification, we obtain $T_{n}=\left\{\cot \left(\theta_{n}-\theta\right)-\cot \left(\theta_{n-1}-\right.\right.$ $\theta)\}$. Note that $\sum_{n=1}^{p} T_{n}=0$, therefore (7) reduces to

$$
k^{2} F(k \cos \theta, k \sin \theta)=\sum_{n=1}^{p} T_{n} e^{-j k\left(a_{n} \cos \theta+b_{n} \sin \theta\right)} .
$$

Note that $T_{n}$ does not depend on the coordinates of the corner and, hence, is independent of the object location. Now, let us shift

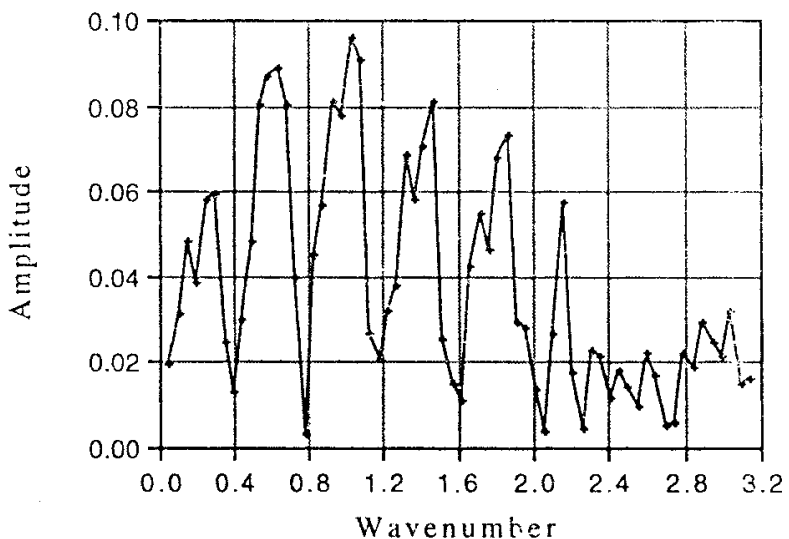

Fig. 5. Computed backscatter from a weakly scattering square object (shown in Fig. 3) illuminated by a broadband signal.

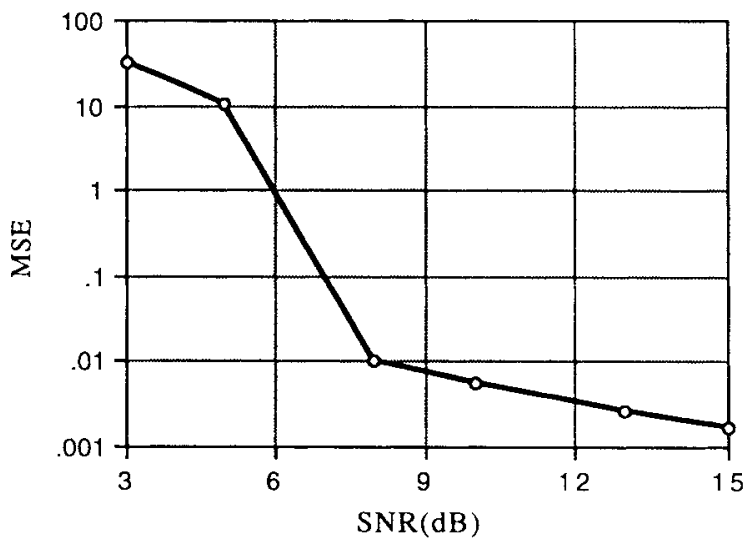

Fig. 6. MSE $\left(m^{2}\right)$ in the estimation of $x$ and $y$ coordinates as a function of SNR.

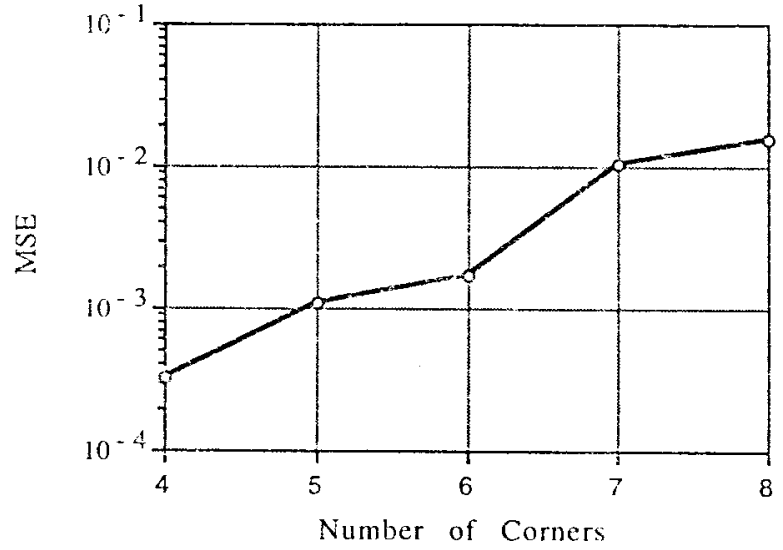

Fig. 7. Mean square error $\left(\mathrm{m}^{2}\right)$ in the estimation of the coordinates of the corners as a function of the number of corners.

the object to a new location, $\left(x_{c}, y_{c}\right)$, such that the center of the coordinates lies outside the object. Then, the corner coordinates of the shifted object, say $\left(g_{n}, h_{n}\right)$ turn out to be $\left(g_{n}, h_{n}\right)=\left(a_{n}+\right.$ $\left.x_{c}, b_{n}+y_{c}\right)$. Following the well-known shift theorem of the Fourier transform (9) may be expressed as follows:

$$
\begin{aligned}
k^{2} & F_{\text {shifted }}(k \cos \theta, k \sin \theta) \\
& =\sum_{n=1}^{p} T_{n} e^{-j k\left(\left(a_{n}+x_{c}\right) \cos \theta+\left(b_{n}+y_{c}\right)+\sin \theta\right) .}
\end{aligned}
$$


From this, it is clear that we can estimate the corner coordinates of even the objects that are away from the origin, using the same procedure. Note that we are interested in estimating $\left(g_{n}, h_{n}\right)$, which can be easily done without any overhead. As side information we also get $T_{n}, n=1,2, \cdots p$ along with the coordinates of the corners, $\left(g_{n}, h_{n}\right)$, from the Prony's algorithm or it's modern versions.

In the process of joining the neighboring corners, it is suggested that $T_{n}$ be computed and compared with the estimated value $T_{n}$ for the corner formed by the lines. A mismatch is an indication of the possibility that the selected edges are incorrect. We have found that this simple idea works well in most cases. An example is used to illustrate how the amplitude of the sinusoids may be used to ascertain the correct object shape. Consider two square objects with their corners as shown in Fig. 4(a). We shall take it that the corners have been estimated from the scattered field as described in Sections III and IV. We need to connect the corners to form convex objects. The number of objects present should be known. In Fig. 4 , we show three out of four possible convex object sets. The amplitude of the sinusoids for all three models are compared with the observed amplitudes (see Table IV). It is seen that the observed amplitudes are indeed closest to those of the true model.

\section{PERformance AnAlysis}

The performance of the object shape estimation procedure proposed here was studied through numerical experiments. For this, we have considered a square object (refractive index contrast equal to 0.01) of size $8 \times 8 \mathrm{~m}^{2}$ and rotated by $9^{\circ}$ with respect to $x$ axis (see Fig. 3). The object was illuminated with a broadband plane wavefront whose wave number is varied from $\frac{\pi}{64}$ to $\pi$ in steps of $\frac{\pi}{64}$ along three directions, namely, $x$-axis, $y$-axis, and a radial direction at an angle $\theta=30^{\circ}$. The backscatter at each wavenumber was computed using the Fourier transform approach described in [11]. A typical example of backscatter caused by $x$-axis illumination is shown in Fig. 5 . To this scattered field, we added white Gaussian noise such that the signal-to-noise ratio (SNR) is equal to a specified figure. Here, the SNR is defined as

$$
\mathrm{SNR}=10 \log _{10} \frac{\text { average scattered energy }}{\text { noise variance }} .
$$

The corners and also the $T_{n}$ 's were estimated using the procedure given in [8]. The mean square error (MSE) in the estimation of coordinates of the corners was studied as a function of SNR. The results, obtained by averaging over fifty independent experiments, are shown in Fig. 6. Notice that MSE rises very rapidly for SNR below $8 \mathrm{~dB}$. This is largely because the projections of two adjacent corners (e.g. $x_{1}$ and $x_{4}$ and $x_{2}$ and $x_{3}$ in Fig. 3 are close to each other; in this example, they are $1.2515 \mathrm{~m}$ apart. For a different orientation, say, at $6^{\circ}$ when the separation becomes $0.8362 \mathrm{~m}$, the MSE rapidly rises for SNR below $15 \mathrm{~dB}$. The estimation error (MSE) also depends upon the number of corners in a polygonal object. We have numerically studied this phenomenon by increasing the number of corners but keeping the object size approximately same (inscribing circle having same radius). Each object was so aligned that the projections of the corners were approximately uniformly spaced. The results are shown in Fig. 7. Finally, we have experimented with a composite object consisting of two squares [shown in Fig. 4(a)] having in all eight corners. All eight corners were correctly estimated with MSE averaged over all eight corners equal to $6.046 e-03 \mathrm{~m}^{2}$ in the presence of background noise $(\mathrm{SNR}=11 \mathrm{~dB})$, and the corners were also joined correctly.

\section{CONCLUSION}

We have shown that the backscatter from a weakly scattering binary object with polygonal cross section can be expressed as a sum of complex sinusoids, from which we can estimate the corner coordinates. All corners of the object can be estimated with as few as three illuminations. An algorithm to correctly pair the estimated coordinates has been developed. When we have more than one object, the task of joining the corners becomes ambiguous. We have used additional information of amplitude of the sinusoids to overcome the ambiguity. Numerical simulations were carried out to demonstrate the robustness of the method in the presence of noise.

\section{ACKNOWLEDGMENT}

The authors thank an anonymous reviewer for bringing to our attention [12] and [13].

\section{REFERENCES}

[1] O. A. Zuniga and R. M. Haralick, "Corner detection using facet model," in Proc. IEEE Int. Conf. Computer Vision and Pattern Recognition, July 1983, pp. 30-37.

[2] L. Kitchen and A. Rosenfeld, "Gray level corner detection," Pattern Recognit. Lett., vol. 1, pp. 95-102, 1982.

[3] K. Rangarajan, M. Shah, and D. V. Brackle, "Optimal corner detector," in Proc. 2nd Int. Conf. Computer Vision, 1988, pp. 90-94.

[4] G. Medioni and Y. Yasumoto, "Corner detection and curve representation using cubic B-splines," Comput. Vis. Graph. Image Processing, vol. 39, pp. 267-278, 1987.

[5] F. Cheng and W. Hsu, "Parallel algorithm for corner finding on digital curves," Pattern Recognit. Lett., vol. 8, pp. 47-53, 1988.

[6] J. S. Lee, Y. N. Sun, C. H. Chen, and C. T. Tsai, "Wavelet based corner detection," Pattern Recognit., vol. 26, pp. 853-865, 1993.

[7] D. W. Tufts and R. Kumaresan, "SVD and improved frequency estimation using linear prediction," IEEE Trans. Acoust., Speech, Signal Processing, vol. 30, pp. 671-675, 1982.

[8] S. Y. Kung, K. S. Arun, and D. V. B. Rao, "State space and singular value decomposition based approximation methods for the harmonic retrieval problem," J. Opt. Soc. Amer., vol. 73, pp. 1799-1811, 1983.

[9] A. J. Devaney, Diffraction Tomography in Inverse Methods in Electromagnetic Imaging Part 2, W. M. Boerner et al., Eds. Amsterdam, The Netherlands: D. Reidel, 1985, pp. 1107-1135.

[10] A. C. Kak, "Tomographic imaging with diffracting and nondiffracting sources," in Array Signal Processing, S. Haykin, Ed. Englewood Cliffs, NJ: Prentice-Hall, 1985, pp. 351-428.

[11] P. S. Naidu, A. Vasuki, P. Satyamurthy, and L. Anand, "Diffraction tomographic imaging with circular array," IEEE Trans. Ultrason., Ferroelect., Freq. Contr., vol. 42, pp. 787-789, 1995.

[12] P. Milanfar, W. C. Karl, and A. S. Willsky, "Reconstructing binary polygonal objects from projections: A statistical view," CVGIP: Graph. Models Image Processing, vol. 56, pp. 371-391, 1994.

[13] P. Milanfar, G. C. Verghese, W. C. Karl, and A. S. Willsky, "Reconstructing polygons from moments with connections to array processing," IEEE Trans. Signal Processing, vol. 43, pp. 432-443, 1995.

[14] P. S. Naidu, Modern Spectrum Analysis of Time Series. Boca Raton, FL: CRC, 1995. 